\title{
Scientific Discovery through Advanced Computing
}

\author{
Carl Edward Oliver \\ Associate Director of \\ Science for the Office of Advanced Scientific Computing Research \\ U. S. Department of Energy, SC-30 \\ 19901 Germantown Road \\ Germantown, Maryland 20874-1290 \\ Phone: +1-(301)-903-7486 \\ FAX: +1-(301)-903-4846 \\ ed.oliver@science.doe.gov
}

\begin{abstract}
Scientific Discovery through Advanced Computing (SciDAC), a new initiative in the Department of Energy's Office of Science, will be described. Computational modeling and simulation are essential to all of the programs in the Office of Science and each of the programs has identified major scientific challenges that can only be addressed through advances in scientific computing.

Advances in computing technologies during the past decade have set the stage for significant advances in modeling and simulation in the coming decade. Several computer vendors promise to increase "peak" performance a 1000-fold in the next five years. Our challenge is to make similar advances in the scientific codes so performance does not degrade as the number of processors increases. This translates to increased investments in algorithms, tools, networking, system software, and applications software. Large interdisciplinary teams of applied mathematicians, computer scientists, and computational scientists are being formed to tackle this daunting problem.

These teams will be supported by a Scientific Computing Hardware Infrastructure designed to meet the needs of the Office of Science's research programs. It will be robust-to provide a reliable source of computing resources for scientific research; agile-to respond to innovative advances in computer technology; and flexible-to ensure that the most effective and efficient resources are used to solver each class of problems.

A status of SciDAC in its initial year and view of where we would like to be in five years will be presented.
\end{abstract}

V.N. Alexandrov et al. (Eds.): ICCS 2001, LNCS 2073, p. 4, 2001.

(C) Springer-Verlag Berlin Heidelberg 2001 